\title{
Tracking Chromosome Evolution in Southern African Gerbils Using Flow-Sorted Chromosome Paints
}

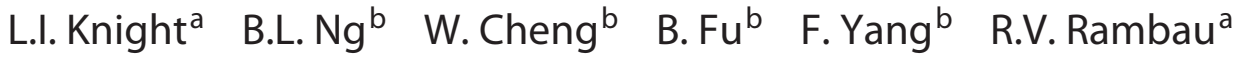 \\ ${ }^{a}$ Evolutionary Genomics Group, Department of Botany and Zoology, University of Stellenbosch, Stellenbosch, South Africa; \\ ${ }^{b}$ Wellcome Trust Sanger Institute, Wellcome Trust Genome Campus, Hinxton, UK
}

\section{Key Words}

Chromosome painting · Gerbillinae - Gerbilliscus paeba .

Robertsonian rearrangements

\begin{abstract}
Desmodillus and Gerbilliscus (formerly Tatera) comprise a monophyletic group of gerbils (subfamily Gerbillinae) which last shared an ancestor approximately 8 million years ago; diploid chromosome number variation among the species ranges from $2 n=36$ to $2 n=50$. In an attempt to shed more light on chromosome evolution and speciation in these rodents, we compared the karyotypes of 7 species, representing 3 genera, based on homology data revealed by chromosome painting with probes derived from flow-sorted chromosomes of the hairy footed gerbil, Gerbillurus paeba $(2 n=$ 36). The fluorescent in situ hybridization data revealed remarkable genome conservation: these species share a high proportion of conserved chromosomes, and differences are due to 10 Robertsonian $(\mathrm{Rb})$ rearrangements ( 3 autapomorphies, 3 synapomorphies and 4 hemiplasies/homoplasies). Our data suggest that chromosome evolution in Desmodillus occurred at a rate of $\sim 1.25$ rearrangements per million years (Myr), and that the rate among Gerbilliscus over a time period spanning $8 \mathrm{Myr}$ is also $\sim 1.25$ rearrangements/Myr. The re-
\end{abstract}

cently diverged Gerbillurus (G. tytonis and G. paeba) share an identical karyotype, while Gerbilliscus kempi, G. afra and G. leucogaster differ by $6 \mathrm{Rb}$ rearrangements (a rate of $\sim 1$ rearrangement/Myr). Thus, our data suggests a very slow rate of chromosomal evolution in Southern African gerbils.

Copyright $\odot 2013$ S. Karger AG, Basel

Southern African gerbils are a monophyletic assemblage of rodents composed of 3 genera and 9 species occurring predominantly in the arid parts of this subregion. They include the monotypic Desmodillus (Desmodillus auricularis) and the polytypic genera Gerbilliscus (G. afra, G. leucogaster, G. brantsii, G. inclusa) and Gerbillurus (G. paeba, G. tytonis, G. vallinus, G. setzeri) [Chimimba and Bennett, 2005; Musser and Carleton, 2005]. The radiation of Gerbillinae spans roughly 10 million years (Myr) with one of the most derived species among Southern African taxa (G. paeba) having diverged $\sim 2.9$ million years ago (Mya) [Chevret and Dobigny, 2005; Colangelo et al., 2007]. Our view of evolutionary relationships among the taxa has fluctuated significantly over the past 2 decades. Initially, Gerbilliscus and Gerbillurus were treated as separate genera [Pavlinov, 2001; Chimimba and Bennett, 2005; Musser and Carleton, 2005]. However, mitochon-

\begin{tabular}{|c|c|}
\hline KARGER & $\begin{array}{l}\text { (๑) } 2013 \text { S. Karger AG, Basel } \\
1424-8581 / 13 / 1394-0267 \$ 0 / 0\end{array}$ \\
\hline $\begin{array}{l}\text { E-Mail karger@karger.com } \\
\text { www.karger.com/cgr }\end{array}$ & $\begin{array}{l}\text { This is an Open Access article licensed under the terms of } \\
\text { the Creative Commons Attribution-NonCommercial } 3.0 \\
\text { License (www.karger.com/OA-license-WT), applicable to } \\
\text { the online version of the article only. Distribution for non- } \\
\text { commercial purposes only. }\end{array}$ \\
\hline
\end{tabular}

Rambau R.V.

Department of Botany and Zoology

University of Stellenbosch

Private Bag X1, Matieland, Stellenbosch 7602 (South Africa)

E-Mail rvr2@sun.ac.za 
drial and nuclear sequence data indicate that some of the lineages within Gerbilliscus cluster with Gerbillurus [Chevret and Dobigny, 2005; Colangelo et al., 2007; Granjon et al., 2012], suggesting a polyphyletic origin for the genera, which in turn prompted the recommendation for Gerbillurus to be considered a synonym of Gerbilliscus. Here, we adopt the name Gerbilliscus (subsuming Gerbillurus) in recognition of the patterns suggested by the robust molecular phylogenies [Colangelo et al., 2007; Granjon et al., 2012]. In effect, this implies that Gerbilliscus contains 3 lineages: an eastern group (G. robusta, G. phillipsi, G. vicinus, G. nigricaudus), a western group (G. gambianus, G. guinea, G. kempi), and a southern group ( $G$. brantsii, G. afra, G. leucogaster), with G. paeba, G. setzeri and $G$. vallinus basal to the western + southern clade.

Diploid chromosome numbers of Southern African gerbils range from $2 \mathrm{n}=36$ (G. paeba) to $2 \mathrm{n}=50$ (D. auricularis) [Qumsiyeh, 1986a, b; Qumsiyeh and Chesser, 1988; present data], and G-band analyses indicate that the variation is due primarily to Robertsonian $(\mathrm{Rb})$ fusions and inversions, with the exception of G. paeba and G. $t y$ tonis which have nearly identical karyotypes [Qumsiyeh et al., 1991; Colangelo et al., 2007]. Intrageneric rearrangements among G. leucogaster, G. afra and G. kempi involve maximally $3 \mathrm{Rb}$ fissions and $3 \mathrm{Rb}$ fusions [Qumsiyeh, 1986b; Volobouev et al., 2007]. Although phylogenetic inferences based on chromosome banding data are valuable [Kovalskaya et al., 2011; Sannier et al., 2011], unequivocal establishment of orthology across taxa may be problematic, and this may hamper detailed descriptions of chromosome rearrangements driving chromosome evolution within taxonomic groups.

Here genome-wide comparisons of the Southern African D. auricularis, G. tytonis, G. leucogaster, G. afra, the West African G. kempi and the North African Psammomys obesus were undertaken in order to refine previous comparisons based on G-banding. In doing so, we aim at providing new insights into chromosomal rearrangements that distinguish these Southern African taxa. This was accomplished using G. paeba whole-chromosome painting probes (reported here for the first time) to identify regions of orthology, and to document genome repatterning among Desmodillus (D. auricularis), Gerbilliscus (G. paeba, G. tytonis, G. leucogaster, G. afra, G. kempi) and the outgroup species, $P$. obesus. Interpreting these differences in the context of the robust DNA sequencebased phylogeny [Chevret and Dobigny, 2005; Colangelo et al., 2005, 2007; Granjon et al., 2012] allowed inferences on the mode and rate of chromosome evolution in Southern African gerbils.

\section{Materials and Methods}

\section{Specimens and Tissue Culture}

Seven species from 3 gerbilline genera were examined. These include 5 Southern African taxa (D. auricularis, G. paeba, G. tytonis, G. leucogaster, and G. afra) as well as G. kempi (West Africa) and P. obesus (North Africa), respectively (table 1). Tissue biopsies (tail or rib muscle) were used to establish fibroblast cultures in DMEM medium (GIBCO) enriched with $15 \%$ foetal calf serum (FCS; GIBCO), amniomax supplement with amniomax basal medium (GIBCO) and Gentamicin $(50 \mu \mathrm{g} / \mathrm{ml})$. Cell cultures were incubated in chambers regulated at $37^{\circ} \mathrm{C}$ and $5 \% \mathrm{CO}_{2}$. Cell division was arrested at metaphase stage using colcemid (with final concentration at $0.10 \mu \mathrm{g} / \mathrm{ml}$; GIBCO) and metaphase cells were harvested using a $0.075 \mathrm{M} \mathrm{KCl}$ hypotonic treatment and subsequent fixation with modified Carnoy's fixative (methanol:glacial acetic acid, 3:1).

\section{Chromosome Banding and Karyotypes}

Chromosome G- and C-banding followed the protocols of Seabright [1971] and Sumner [1972], respectively. Briefly, Gbanding of metaphase chromosome spreads required aging the slides for a minimum of $1 \mathrm{~h}$ at $65^{\circ} \mathrm{C}$ and treating aged slides with a trypsin solution $(0.25 \%$ in $1 \times$ PBS $)$ for a minimum of 30 s. Trypsin-treated slides were rinsed in calf serum buffer (containing 500 $\mu \mathrm{l} \mathrm{FCS}$ and $50 \mathrm{ml} 0.025 \mathrm{M} \mathrm{KH}_{2} \mathrm{PO}_{4}$ buffer, $\mathrm{pH}$ 6.8) for $3 \mathrm{~min}$ followed by Giemsa staining (made in $0.025 \mathrm{M} \mathrm{KH}_{2} \mathrm{PO}_{4}$ buffer; $\mathrm{pH}$ 6.8 ) to visualise chromosomes. C-banding entailed immersing slides in a $0.2 \mathrm{M} \mathrm{HCl}$ solution for $3 \mathrm{~min}$, followed by incubation in a saturated $\mathrm{Ba}(\mathrm{OH})_{2}$ solution for a minimum of $70 \mathrm{~s}$ at $55^{\circ} \mathrm{C}$. A second incubation in $2 \times \mathrm{SSC}$ for $30-60 \mathrm{~min}$ at $50-60^{\circ} \mathrm{C}$ (speciesdependent) followed. Chromosomes were visualised using $2 \% \mathrm{Gi}-$ emsa staining and arranged according to karyotypes published by Qumsiyeh [1986a, b], Qumsiyeh and Chesser [1988] and Volobouev et al. [2007].

\section{Flow-Sorting and Fluorescent in situ Hybridization}

G. paeba $(2 \mathrm{n}=36)$ was selected for flow-sorting primarily because it is a terminal taxon among the Southern African species in the molecular phylogeny [see Chevret and Dobigny, 2005]. Chromosomes were sorted on a MoFlo dual-laser cell sorter and isolated on size and base-pair composition [Yang et al., 2009]. Biotin16-dUTP- (Roche) or digoxigenin-11-dUTP-labelled painting probes were made by DOP-PCR amplification of flow-sorted chromosomes [Telenius et al., 1992]. Fluorescent in situ hybridization (FISH) experiments followed Rens et al. [2006] and Yang and Graphodatsky [2009] with minor modifications. Biotin-labelled probes were detected with the antibody Cy3-streptavidin (1:500 dilution), and digoxigenin-labelled probes with FITC-conjugated anti-digoxigenin IgG made in sheep $(1: 1,000)$. Slides were counterstained with $0.1 \mu \mathrm{g} / \mathrm{ml}$ DAPI (4',6-diamidino-2-phenylindole) and mounted using Vectashield medium (Vector Labs). Images were captured using a CCD camera attached to an Olympus BX60 epifluorescence microscope equipped with DAPI, FITC and Cy3 fluorescence filters. Images were edited using the Genus software system (Applied Imaging Corp., Newcastle, UK).

\section{Consensus Phylogeny}

A consensus phylogenetic tree that includes the species under examination (i.e. D. auricularis, G. paeba, G. tytonis, G. leucogaster, G. afra, G. kempi, and P. obesus) was obtained from the more 
Table 1. Specimens examined in the study and their respective localities in South Africa (RSA), Namibia, West and North Africa

\begin{tabular}{|c|c|c|c|c|c|}
\hline Species & Locality & Map coordinates & Sex & $2 n$ & Specimen (n) \\
\hline Desmodillus auricularis & $\begin{array}{l}\text { Henkries, Northern Cape, RSA } \\
\text { Tankwa Karoo, Northern Cape, RSA } \\
\text { Three Sisters, Northern Cape, RSA }\end{array}$ & $\begin{array}{l}28^{\circ} 57^{\prime} 57^{\prime \prime} \mathrm{S}, 18^{\circ} 08^{\prime} 54^{\prime \prime} \mathrm{E} \\
32^{\circ} 12^{\prime} 19^{\prime \prime} \mathrm{S}, 19^{\circ} 09^{\prime} 10^{\prime \prime} \mathrm{E} \\
31^{\circ} 53^{\prime} 14^{\prime \prime} \mathrm{S}, 23^{\circ} 05^{\prime} 17^{\prime \prime} \mathrm{E}\end{array}$ & $\begin{array}{l}\lambda \\
0 \\
0 \\
0\end{array}$ & $\begin{array}{l}50 \\
50 \\
50\end{array}$ & $\begin{array}{l}1 \\
2 \\
1\end{array}$ \\
\hline Gerbilliscus kempi & Senegal & - & q & 48 & 1 \\
\hline Gerbilliscus afra & $\begin{array}{l}\text { Clanwilliam, Western Cape, RSA } \\
\text { Clanwilliam, Western Cape, RSA } \\
\text { Vensterklip, Western Cape, RSA }\end{array}$ & $\begin{array}{l}32^{\circ} 10^{\prime} 42^{\prime \prime} \mathrm{S}, 18^{\circ} 53^{\prime} 27^{\prime \prime} \mathrm{E} \\
32^{\circ} 10^{\prime} 42^{\prime \prime} \mathrm{S}, 18^{\circ} 53^{\prime} 27^{\prime \prime} \mathrm{E} \\
32^{\circ} 18^{\prime} 56^{\prime \prime} \mathrm{S}, 18^{\circ} 23^{\prime} 46^{\prime \prime} \mathrm{E}\end{array}$ & $\begin{array}{l}\hat{O} \\
+ \\
+ \\
+\end{array}$ & $\begin{array}{l}44 \\
44 \\
44\end{array}$ & $\begin{array}{l}1 \\
1 \\
3\end{array}$ \\
\hline Gerbilliscus leucogaster & $\begin{array}{l}\text { Windhoek, Namibia } \\
\text { Windhoek, Namibia }\end{array}$ & $\begin{array}{l}22^{\circ} 33^{\prime} 34^{\prime \prime} \mathrm{S}, 17^{\circ} 05^{\prime} 56^{\prime \prime} \mathrm{E} \\
22^{\circ} 33^{\prime} 34^{\prime \prime} \mathrm{S}, 17^{\circ} 05^{\prime} 56^{\prime \prime} \mathrm{E}\end{array}$ & $\begin{array}{l}\hat{0} \\
+ \\
+\end{array}$ & $\begin{array}{l}40 \\
40\end{array}$ & $\begin{array}{l}1 \\
1\end{array}$ \\
\hline Gerbilliscus paeba & $\begin{array}{l}\text { Anysberg NR, Northern Cape, RSA } \\
\text { Sutherland, Northern Cape, RSA } \\
\text { Swakopmund, Namibia } \\
\text { Swakopmund, Namibia } \\
\text { Three Sisters, Northern Cape, RSA }\end{array}$ & $\begin{array}{l}33^{\circ} 23^{\prime} 20^{\prime \prime} \mathrm{S}, 20^{\circ} 39^{\prime} 20^{\prime \prime} \mathrm{E} \\
33^{\circ} 28^{\prime} 48^{\prime \prime} \mathrm{S}, 20^{\circ} 36^{\prime} 20^{\prime \prime} \mathrm{E} \\
22^{\circ} 40^{\prime} 36^{\prime \prime} \mathrm{S}, 14^{\circ} 31^{\prime} 35^{\prime \prime} \mathrm{E} \\
22^{\circ} 40^{\prime} 36^{\prime \prime} \mathrm{S}, 14^{\circ} 31^{\prime} 35^{\prime \prime} \mathrm{E} \\
31^{\circ} 53^{\prime} 14^{\prime \prime} \mathrm{S}, 23^{\circ} 05^{\prime} 17^{\prime \prime} \mathrm{E}\end{array}$ & $\begin{array}{l}\hat{0} \\
\hat{0} \\
\hat{0} \\
\phi \\
+ \\
\hat{0}\end{array}$ & $\begin{array}{l}36 \\
36 \\
36 \\
36 \\
36\end{array}$ & $\begin{array}{l}1 \\
3 \\
1 \\
1 \\
1\end{array}$ \\
\hline Psammomys obesus & Tunisia & - & q & 48 & 1 \\
\hline
\end{tabular}

comprehensive DNA-based phylogenies of Dobigny and Chevret [2005], Colangelo et al. [2005, 2007] and Granjon et al. [2012]. In effect, our tree emphasises the monophyly of Gerbilliscus + Gerbillurus (both referred to as Gerbilliscus), the basal position of Desmodillus to the Southern African taxa, and the clustering of $P$. obesus outside the Southern African taxa, all well-established evolutionary associations. The latter species was used to polarize some of the chromosomal changes observed at the base of Southern African taxa.

\section{Results and Discussion}

\section{Characterisation of the G. paeba Flow Karyotype and Cross-Species FISH}

The 36 chromosomes of $G$. paeba (GPA; $2 \mathrm{n}=36)$ were resolved into 16 peaks, of which 14 each contained a single type of autosome $(1,4-6,7-8,12-17, \mathrm{X}, \mathrm{Y}), 1$ peak contained 2 autosomes $(2+3)$ and 1 contained 4 autosomes $(7+9+10+11$; fig. 1$)$. The homologues of GPA7, which are $\mathrm{C}$-positive, were sorted into 2 peaks, likely as a result of variation in the amount of heterochromatin.

G. paeba chromosome paints were successfully hybridized onto metaphase chromosomes of all gerbil taxa analyzed, enabling detailed comparisons among them (figs. 2-5; table 2). Together with banding comparisons, the data from chromosome painting show slight differences in genome conservation among the species. Nine-

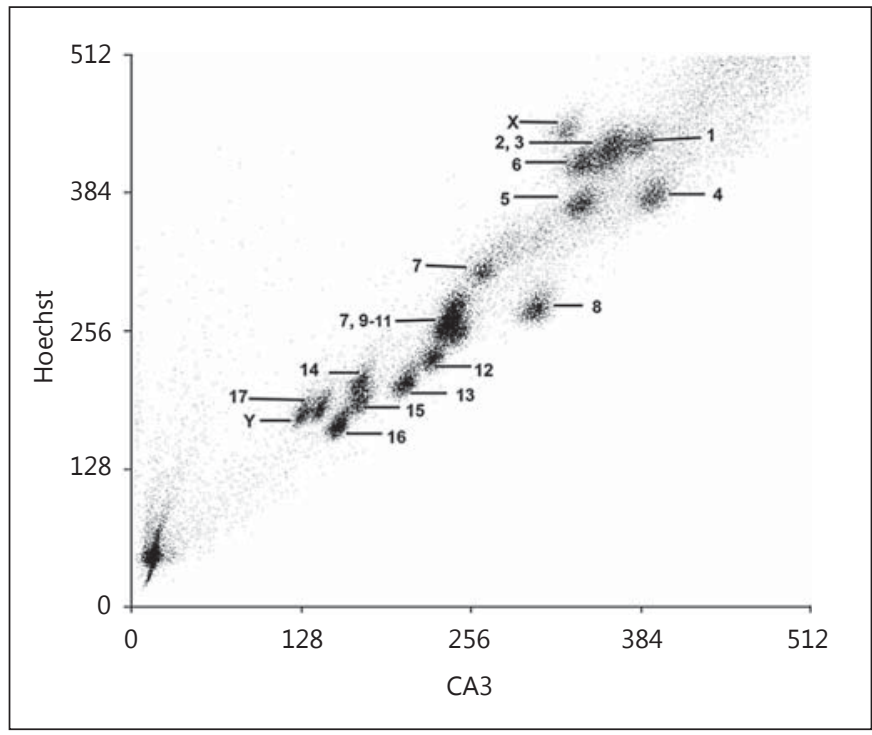

Fig. 1. Bivariate flow karyotype of a male fibroblast cell line from G. paeba $(2 \mathrm{n}=36)$.

teen homologous regions were identified in G. tytonis (GTY; fig. 2) - all chromosomes were conserved intact (correspondence of the probe containing multiple chromosomes was verified using G-bands of G. paeba). The same set of chromosome paints delimited 21 synteny- 


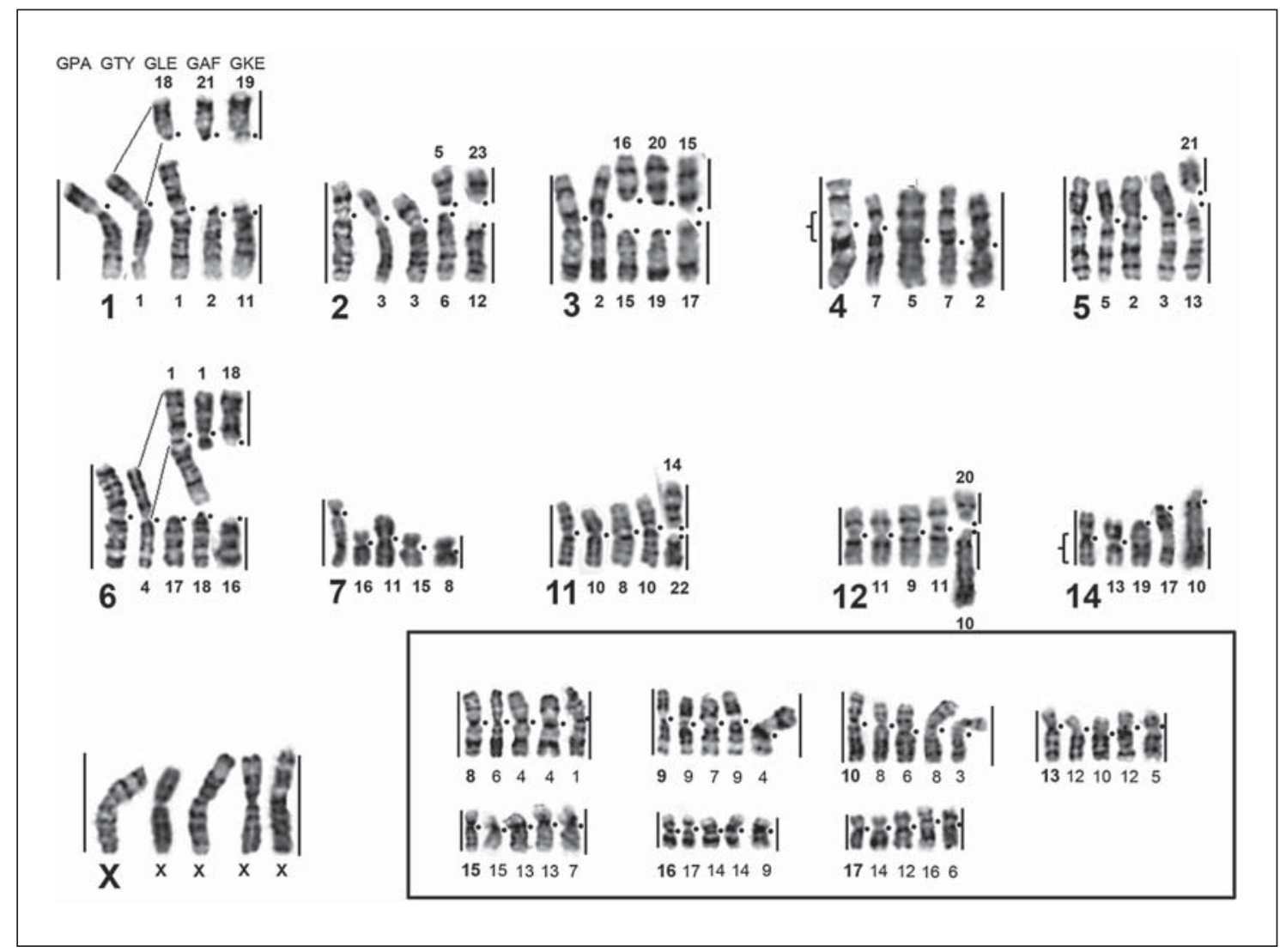

Fig. 2. Partial homology map among G. paeba (GPA), G. tytonis (GTY), G. leucogaster (GLE), G. afra (GAF), and G. kempi (GKE) based on chromosome painting and G-banding, with GPA chromosomes as references. Chromosomes that were conserved in their entirety in all Gerbilliscus taxa are in the inset. The regions of homology are indicated by the vertical lines and GPA chromosome numbers (large font). Centromere positions are indicated by closed circles, and the brackets indicate the boundaries of inversions. Chromosome numbers correspond to those in the respective species' karyotypes.

Table 2. Presence (+) and absence (-) of Robertsonian rearrangements in the 7 gerbil species analysed

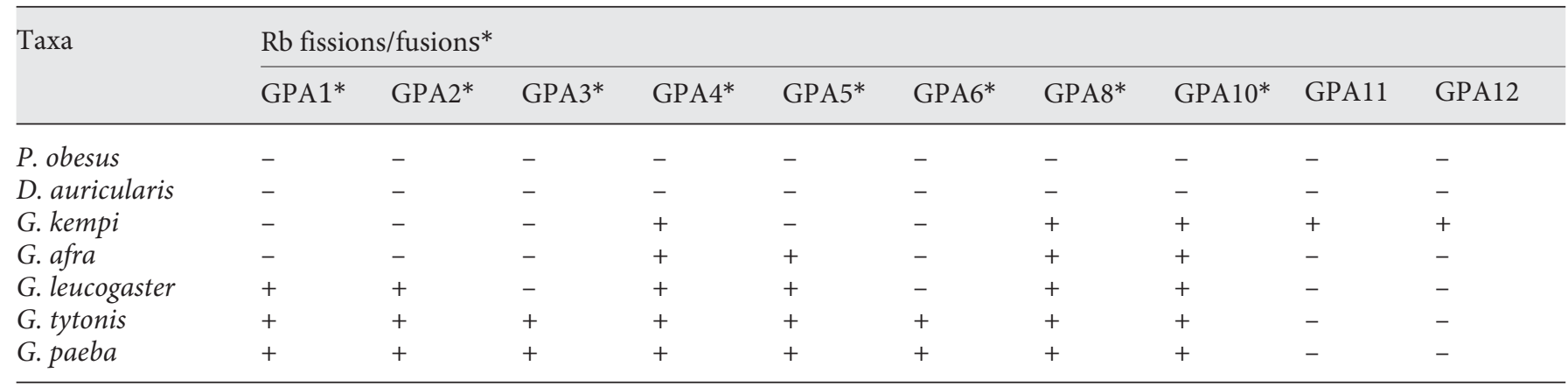

Chromosome numbers correspond to G. paeba (GPA), the species used for flow-sorting the painting probes. Robertsonian fusions are indicated by an asterisk. 


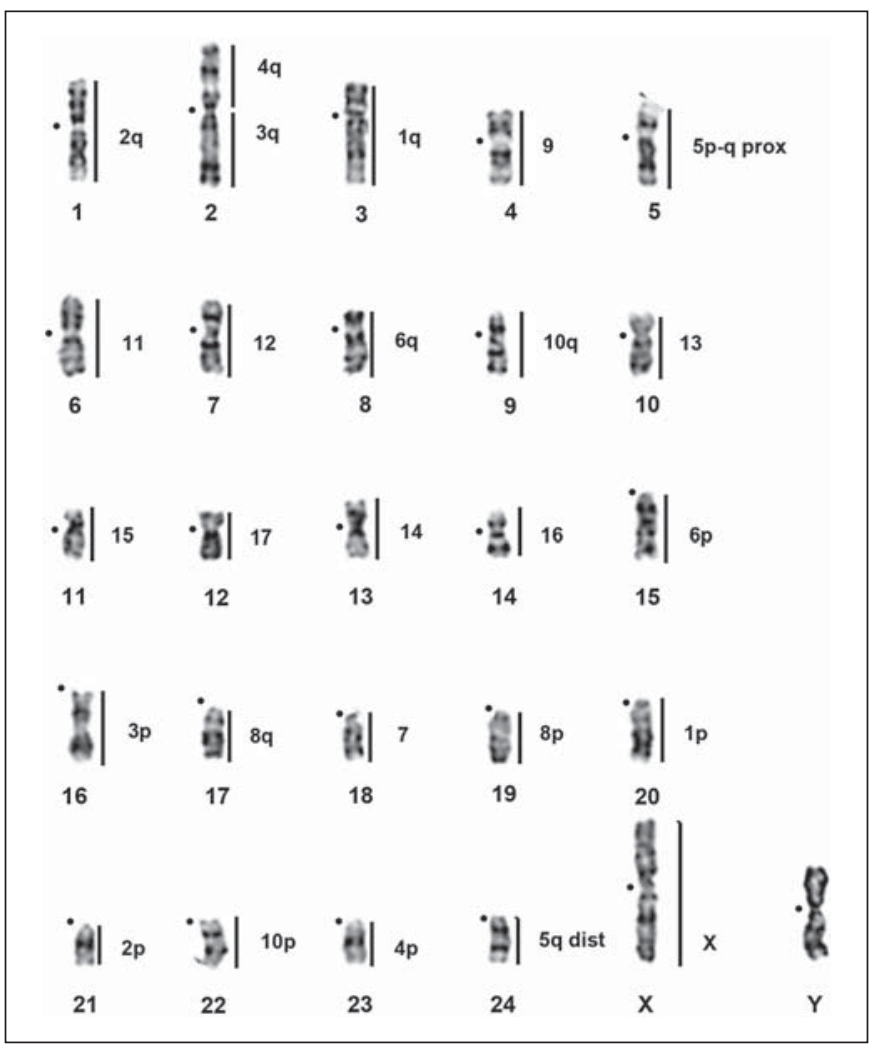

Fig. 3. G-banded haploid karyotype of a male $D$. auricularis with regions of orthology to G. paeba indicated by vertical lines (numbered to the right) as determined by cross-species chromosome painting. Centromere positions are indicated by closed circles.

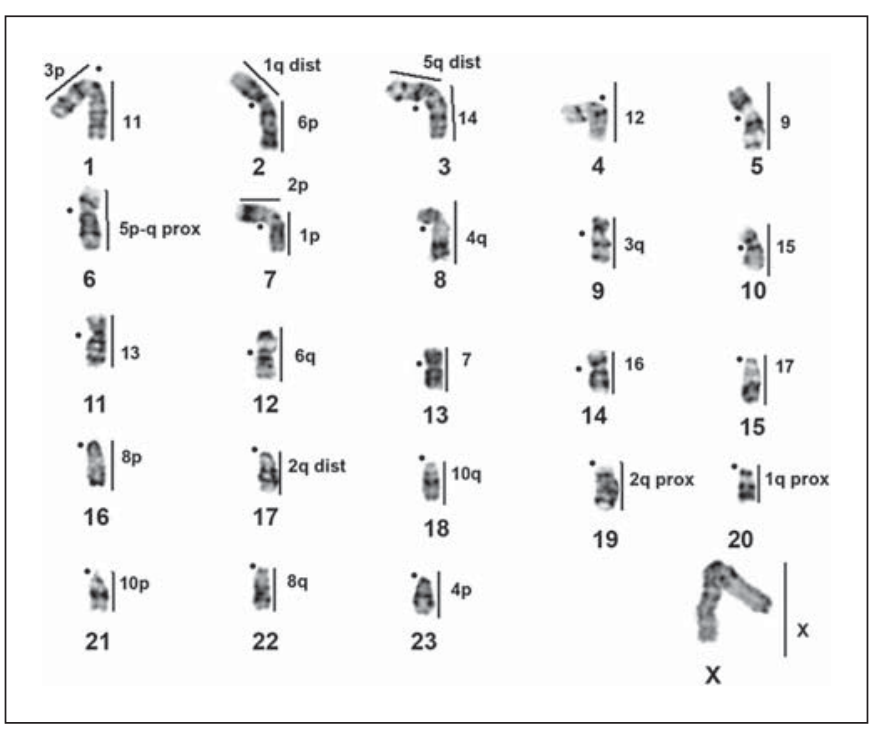

Fig. 4. G-banded haploid karyotype of a male P. obesus with regions of orthology to G. paeba indicated by vertical lines (numbered to the right) as determined by cross-species chromosome painting. Centromere positions are indicated by closed circles.

Comparative Chromosome Painting in Gerbils conserved regions in G. leucogaster (GLE), 22 regions in G. afra (GAF) (fig. 2) and 25 in G. kempi (GKE) (fig. 2). In these 3 species (G. leucogaster, G. afra, G. kempi), 14 to 15 GPA chromosomes (GPA4-17 and the X) are each retained as a single chromosome, and 3 GPA chromosomes (GPA1, 3, 6) each correspond to 2 chromosomes. Furthermore, GPA2 probe painted 2 segments in G. afra and G. kempi, while GPA5 and 12 produced 2 signals in $G$. kempi (fig. 2). Cross-species chromosome painting onto metaphases of $D$. auricularis (DAU) revealed 26 regions of homology (fig. 3). Of these, 10 were conserved as single chromosomes homologous to GPA7, 9, 11-17 and the X, and 8 were conserved as 2 chromosomes, or chromosome segments, corresponding to GPA1-6, 8 and 10 (fig. 3). Lastly, 28 homologous regions were detected in P. obesus (POB), wherein 10 regions each correspond to $1 \mathrm{GPA}$ chromosome (GPA7, 9, 11-17 and X), 6 GPA chromosomes (GPA3-6, 8 and 10) each are homologous to $2 \mathrm{POB}$ chromosomal fragments, and 2 (GPA1 and 2) each are homologous to 3 POB fragments (table 2; fig. 4). The level of genome conservation is consistent with previously established evolutionary relations among these species: Desmodillus is basal in the phylogeny, while the Gerbilliscus taxa form a monophyletic clade with G. paeba and G. tytonis possessing identical karyotypes [Chevret and Dobigny, 2005; Colangelo et al., 2007; Granjon et al., 2012].

Discrepancies between G-Banding and FISH Results

While the FISH results were largely in agreement with published data based on G-band comparison [Qumsiyeh, 1986a, b; Qumsiyeh and Chesser, 1988; Qumsiyeh et al., 1991; Colangelo et al., 2001; Volobouev et al., 2007], we were able to correct several mismatches in the identification of presumptive homologues and resolve some of the problematic associations reported in previous comparisons. This is most evident in the comparison between $G$. kempi and G. tytonis [Volobouev et al., 2007] as well as between Desmodillus and G. paeba [Qumsiyeh, 1986a, b]. Banding analyses initially suggested GTY1, 2, 3, 5, 10 and 11 to be homologous to GKE17 + 11, $15+14,12+20,13$ $+19,21+22$ and $23+10$ prox, respectively [Volobouev et al., 2007]. Our data unequivocally identified GTY1 = GKE11 + 19, GTY2 = GKE15 + 17, GTY3 = $12+23$, GTY5 = GKE13 + 21, GTY10 = GKE14 + 22, and GTY11 = GKE20 + 10prox (fig. 2). Secondly, DAU2 was initially described as homologous to GPA3q plus a de novo euchromatic addition [Qumsiyeh, 1986b]. Our results indicate that the 'euchromatic addition' is in fact homologous to GPA4q (fig. 3). 
Fig. 5. Examples of FISH of G. paeba (GPA) painting probes onto $G$. tytonis (GTY), G. kempi (GKE), D. auricularis (DAU), and $P$. obesus (POB). a Hybridization of GPA14 (Cy3) on GTY13. b Hybridization of GPA12 and GPA14 on GKE10proximal and 20, and GKE10distal, respectively. c FISH of GPA5 on DAU5 and 24. d FISH of GPA1 and GPA6 on POB2p,7q, and 20 and POB2q and 12, respectively. Chromosomes are counterstained with DAPI.
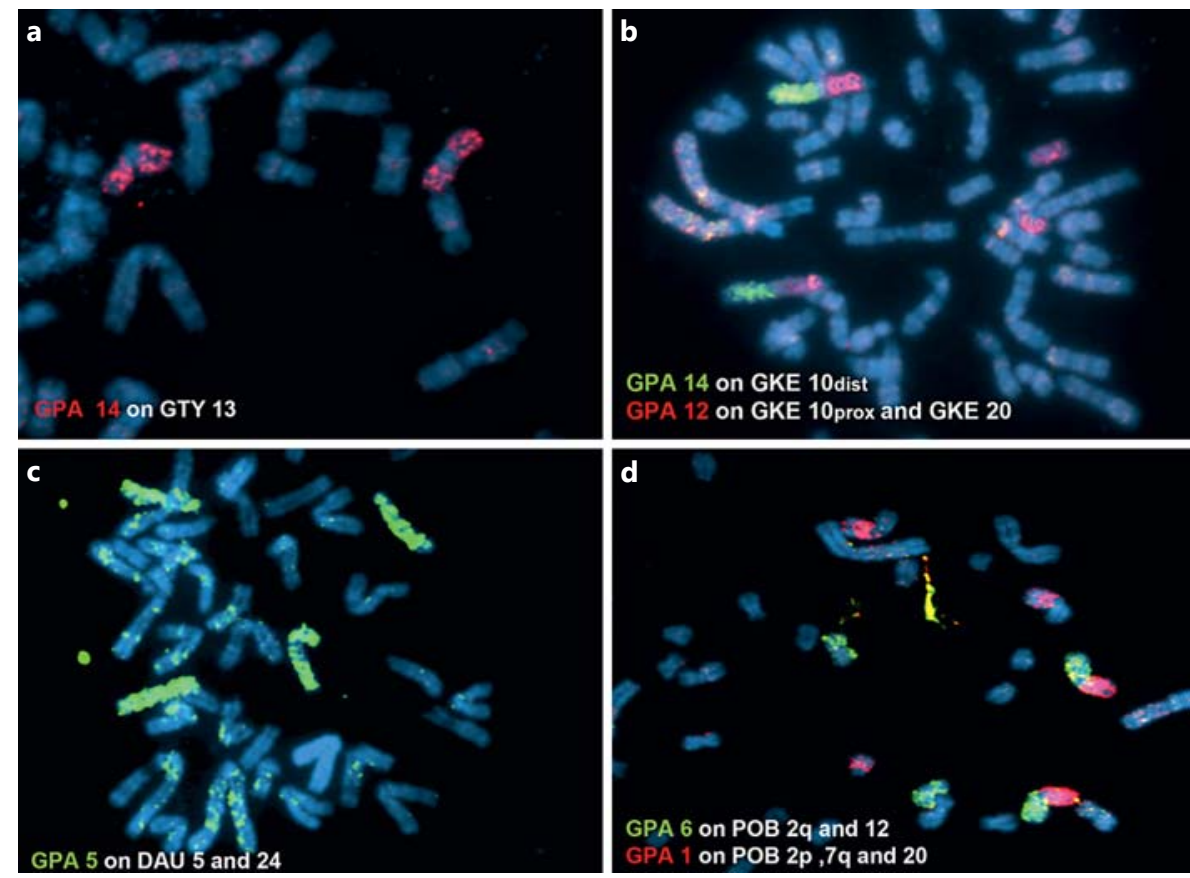

Further, the so-called late replicating GPA7 [Qumsiyeh et al., 1991] successfully hybridized to all species analysed here and was retained as a single chromosome in all species (figs. 2-4). The differences among the chromosomes painted by GPA7 are their sizes, and, in one instance in Desmodillus (DAU18, fig. 3), the difference is due to a pericentric inversion resulting in an acrocentric form (in contrast, all GPA7 homologues in other gerbils are biarmed). Although previous banding comparison suggested GPA7 is wholly heterochromatic [Qumsiyeh, 1988; Qumsiyeh et al., 1991], our data refutes this, especially given the fact that it successfully hybridized to euchromatic genomic fractions in all related species, taxa that last shared an ancestor approximately $9 \mathrm{Mya}$ [Chevret and Dobigny, 2005].

Cross-species chromosome painting also revealed a striking resemblance between karyotypes of G. paeba and G. tytonis, confirming previous banding studies. All GPA chromosomes, including the $\mathrm{Y}$ chromosome, were retained in G. tytonis. In addition, the 2 species are very similar in other respects, notwithstanding their sympatric distribution in Namibia. Other aspects identical between the 2 species include quadrupedal saltation (adaptation for locomotion in sand), absence of sexual dimorphism, nocturnal activity and omnivorous diet [Griffin, 1990; Perrin et al., 1999a, b]. The overlap between the taxa is considerable. For example, G. paeba from the Namib
Desert weighs on average $26.40 \mathrm{~g}$ (20-37 g), has a slightly tufted tail tip, and the hind foot is less than $30 \mathrm{~mm}(21-30$ $\mathrm{mm}$ ), while G. tytonis has an average mass of $24 \mathrm{~g}$ (maximum $35 \mathrm{~g}$ ), a more pronounced, tufted tail, and narrow, hairy feet with hind foot length averaging $33.3 \mathrm{~mm}$ (28$36 \mathrm{~mm}$ ) [Griffin, 1990; Perrin et al., 1999a, b; Chimimba and Bennett, 2005]. Such a pronounced overlap in morphology and habitat preference coupled to their invariant karyotypes suggests a recent divergence and further investigations may show them to be the same species.

\section{Chromosomal Rearrangements and Homoplasy}

Our chromosomal homology map established by FISH and banding comparison demonstrates that $\mathrm{Rb}$ rearrangements were important in chromosomal evolution and speciation in these gerbils (figs. 2, 6). Early G-band comparisons among gerbils have suggested that $\mathrm{Rb}$ rearrangements are homoplasic, and interpreting them in an evolutionary context may be problematic [Qumsiyeh et al., 1987] - a finding recently shown for Bovidae [Robinson and Ropiquet, 2011]. Using the gerbil sequence-based phylogeny as scaffold (fig. 6), we show that of the 10 rearrangements (table 2) identified by the GPA painting probes $1-6,8,10,11$, and 12 , one is an asynapomorphy (GPA6) uniting G. paeba + G. tytonis, 2 are autapomorphies (GPA11 and 12) in G. kempi, 3 are synapomorphies (GPA4, 8 and 10) for all Gerbilliscus, and the remainder 
Fig. 6. A consensus tree derived from mtDNA and nuclear sequence data [Chevret and Dobigny, 2005; Colangelo et al., 2005, 2007; Granjon et al., 2012]. The diploid numbers and the approximate divergence dates were obtained from Colangelo et al. [2007]. Node A is characterised by gross chromosomal homeologies in all species identified with GPA7, 9, 13, 15-17, and the syntenic association GPA4q/3q defines split of $D$. auricularis at node $B$. The nodes $\mathrm{C}-\mathrm{F}$ contain 10 Robertsonian rearrangements of which 4 (involving GPA1, 2, $3,5)$ were characterised as hemiplasies or homoplasies [Avise and Robinson, 2008]. For detailed discussion see text and table 2 .

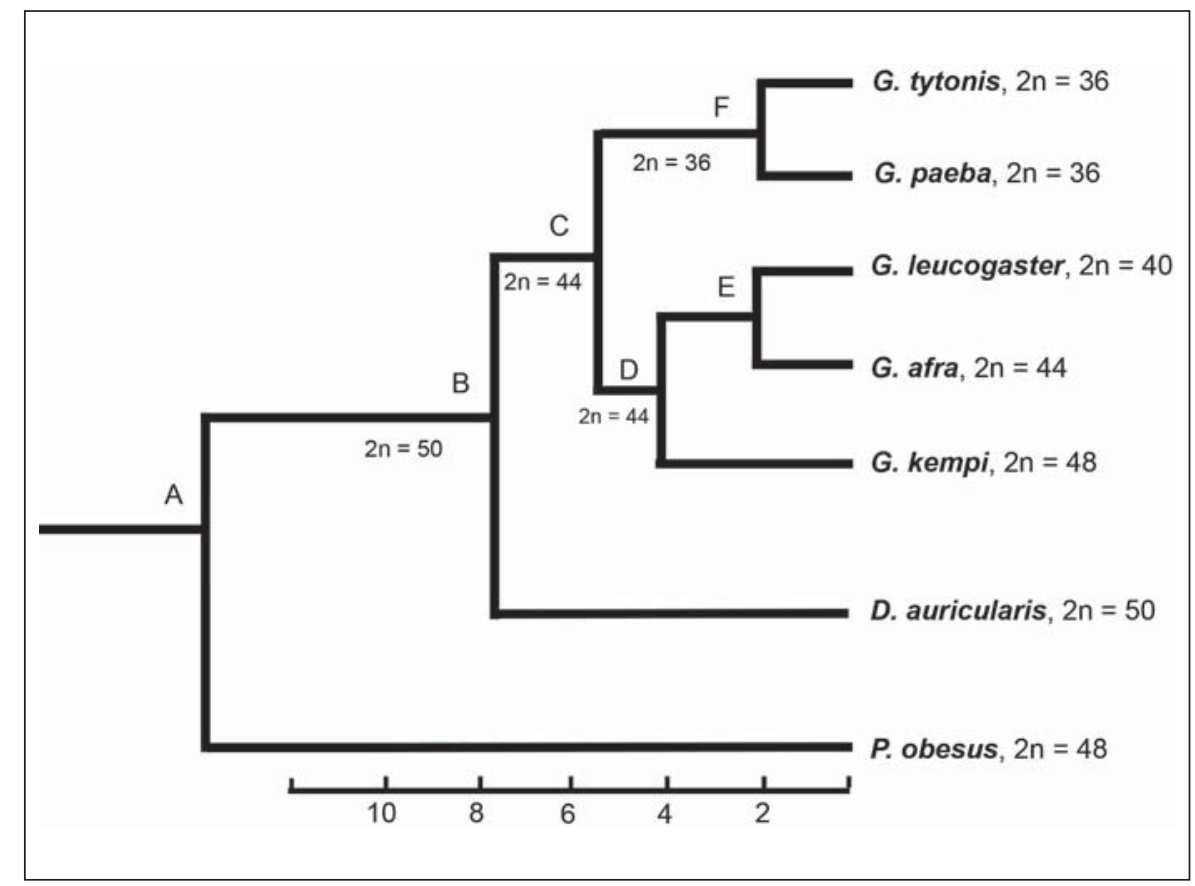

(GPA1, 2, 3, 5) are potential homoplasies/hemiplasies [Avise and Robinson, 2008].

With respect to the first of these, GPA1, 2 possible hypotheses may be suggested for the observed patterns when the character is mapped to the tree in figure 6 . The first is that it is an example of hemiplasy, having undergone a fusion at $\sim 6 \mathrm{Mya}$ (node $\mathrm{C}$ ) and persisted as a polymorphism until the divergence of $G$. leucogaster and G. afra at $\sim 2.5$ Mya. The alternate explanation (i.e. homoplasy) suggests that the fusion arose at node $\mathrm{F}$ (the common ancestor to G. tytonis and G. paeba) and convergently so in $G$. leucogaster (i.e. requiring 2 changes vs. the one for hemiplasy). Although hemiplasy offers the most parsimonious solution in terms of rare genomic changes [Rokas and Holland, 2000], the required persistence time ( 3.5 Myr) is at the upper bound suggested by Robinson et al. [2008] and Robinson and Ropiquet [2011], and we regard homoplasy as the more likely of the 2 hypotheses. The same reasoning applies to the $\mathrm{Rb}$ rearrangements involving GPA2 and GPA3. A more convincing case for hemiplasy can be made with respect to GPA5 (arose at node $\mathrm{C}$ and persisted as a polymorphism for $\sim 2 \mathrm{Myr}$ to $\mathrm{D}$, at which point it was lost in the lineage leading to $G$. kempi).

Consequently, the finding of Qumsiyeh et al. [1987] that homoplasy is present in the chromosomal evolution of gerbils is confirmed by the present study. Our results show that 4 of the 10 rearrangements identified are homoplasic when mapped to the tree (3 convergences/reversals and 1 probable hemiplasy, all of which contribute to the 'noise' evident in the species phylogeny). Qumsiyeh et al. [1987] on the other hand obtained 7 homoplasies in $15 \mathrm{Rb}$ rearrangements among G. paeba, G. vallinus, G. robustus, G. nigricauda, G. leucogaster, G. afra, and G. brantsii. It should be noted, however, that the Qumsiyeh et al. [1987] study may have overestimated the number of homoplasic traits due to topological differences in the trees used (allozyme-based in Qumsiyeh et al. [1987], and $\mathrm{mtDNA}$ and nuclear sequences in our case) and, in some instances, to their misidentification of the chromosomes involved.

In addition to the overwhelming presence of $\mathrm{Rb}$ rearrangements in these gerbils, inversion differences were also noted in several species. Side-by-side G-band comparison (directed by FISH) in Desmodillus and G. afra show that 5 autosomal pairs differ due to the position of the centromeres (DAU1 vs. GAF6, DAU3 vs. GAF2, DAU13 vs. GAF17, DAU8 vs. GAF18, DAU18 vs. GAF15; figs. 2, 3). Similarly, 2 GPA autosomes differ from their orthologs in G. afra as a result of centromere variability (GAF17 vs. GPA14 and GAF7 vs. GPA4; online suppl. fig. 1, www.karger.com/doi/10.1159/000350696). Similar observations have been documented for other gerbil species [Volobouev et al., 2007]. 


\section{Ancestral Karyotype Inference}

The use of the DNA sequence phylogeny and the inclusion of $P$. obesus in the comparisons allow us to propose an ancestral karyotype for the last common ancestor to Desmodillus and Gerbilliscus. We hypothesise that this comprised 7 biarmed autosomes which remained unchanged among all the taxa (GPA7, 9, 13, 15, 16, 17, X; table 2), and 21 acrocentrics (GPA1p, 1q, 2p, 2q, 3p, 3q, $4 \mathrm{p}, 4 \mathrm{q}, 5 \mathrm{p}, 5 \mathrm{q}, 6 \mathrm{p}, 6 \mathrm{q}, 8 \mathrm{p}, 8 \mathrm{q}, 10 \mathrm{p}, 10 \mathrm{q}, 11 \mathrm{p}, 11 \mathrm{q}, 12 \mathrm{p}, 12 \mathrm{q}$ and 14). Further refinements to this karyotype can be expected since Psammomys is only 1 of 3 legitimate outgroup taxa (Brachiones, Desmodilliscus, Pachyuromus) to the Southern African gerbil species.

\section{Rate of Chromosome Evolution}

Interpretation of the rearrangements in the context of DNA sequence divergence times allows us to make inferences about the rate of evolution. Desmodillus accumulated $4 \mathrm{Rb}$ rearrangements and 5 inversions since it separated from Gerbilliscus, reflecting a rate of 1.25 rearrangements/Myr (even taking the homoplasic rearrangement detected by GPA5 into account); G. kempi accumulated $3 \mathrm{Rb}$ rearrangements following its split from the G. afra/G. leucogaster lineage 5.5 Mya, which translates into an evolutionary rate of less than 1 rearrangement/Myr. The 2 rearrangements separating $G$. leucogaster from G. afra accumulated over 2.5 Mya, representing a rate of approximately 1 rearrangement/Myr. On average, the rate of chromosomal evolution among Gerbilliscus over $8 \mathrm{Myr}$ is $~ 1.25$ rearrangement/Myr (10 rearrangements over $8 \mathrm{Myr}$ ), which is considerably slower than values previously obtained in other gerbils (e.g. West African Taterillus have a rate of 45 rearrangements/Myr) [Dobigny et al., $2002,2005]$. Some Gerbillus species show a rate of $\sim 12.3$ rearrangements/Myr [Aniskin et al., 2006; Chevret and Dobigny, 2005]. Recently, it was shown that 6 representa- tive genera in the Rattini tribe displayed an evolutionary rate of 0.6-3.33 rearrangements/Myr, which was considered slow for murid rodents [Badenhorst et al., 2011]. Therefore, the rate of chromosome evolution in the Southern African gerbils studied here is unexpectedly slow.

In summary, we report the first genome-wide crossspecies chromosome painting in Southern African gerbilline rodents. The FISH data unequivocally establish homology among taxa from Desmodillus and Gerbilliscus which last shared an ancestor approximately 8 Mya. Our data also corrected some errors in published homeology maps that were based on banding results alone, and thus underscores the utility of FISH for inferring homology between species. Significantly, the present data demonstrates that the rate of chromosome evolution in Gerbilliscus is relatively slow in comparison to other gerbil lineages, which suggests evolutionary rate heterogeneity within these rodents. A more complete Zoo-FISH analysis including more representatives of the 15 genera comprising the subfamily Gerbillinae should provide more detailed assessment of chromosome evolution among these rodents.

\section{Acknowledgements}

Ethical clearance for this project was approved by Sub Committee B of the University of Stellenbosch (Ethics clearance certificate No. 10NP_KNI01). This study was supported by the South African National Research Foundation (to L.I.K. and R.V.R.) and Wellcome Trust (grant number WT098051) to B.L.N., W.C., B.F. and F.Y. The authors thank Drs L. Granjon, Dr. T Wassennar, G. Dobigny and Phillipe Gauthier and Profs. V. Volobouev and K. Bâ for specimens collected outside South Africa. Prof. T.J. Robinson and Dr. G. Dobigny are thanked for comments on earlier drafts of the manuscripts, and 2 anonymous reviewers for the insightful comments.

\section{References}

Aniskin VM, Benazzou T, Biltueva L, Dobigny G, Granjon L, Volobouev V: Unusually extensive karyotype reorganization in four congeneric Gerbillus species (Muridae: Gerbillinae). Cytogenet Genome Res 112:131-140 (2006).

-Avise JC, Robinson TJ: Hemiplasy: a new term in the lexicon of phylogenetics. Syst Biol 57:503507 (2008).

- Badenhorst D, Dobigny G, Adega F, Chaves R, O'Brien PCM, et al: Chromosomal evolution in Rattini (Muridae, Rodentia). Chromosome Res 19:709-727 (2011).
Chevret P, Dobigny G: Systematics and evolution of the subfamily Gerbillinae (Mammalia, Rodentia, Muridae). Mol Phylogenet Evol 35: 674-688 (2005).

Chimimba CT, Bennett NC: Order Rodentia; in Skinner JD, Chimimba CT (eds): The Mammals of the Southern African Subregion, pp 77-209 (Cambridge University Press, Cape Town 2005).

Colangelo P, Civitelli MV, Capanna E: Morphology and chromosomes of Tatera Lataste 1882 (Rodentia Muridae Gerbillinae) in West Africa. Trop Zool 14:243-253 (2001).
Colangelo P, Corti M, Verheyen E, Annesi F, Oguge N, et al: Mitochondrial phylogeny reveals differential modes of chromosome evolution in the genus Tatera (Rodentia: Gerbillinae) in Africa. Mol Phylogenet Evol 35:556568 (2005)

Colangelo P, Granjon L, Taylor PJ, Corti M: Evolutionary systematics in African gerbilline rodents of the genus Gerbilliscus: inference from mitochondrial genes. Mol Phylogenet Evol 42:797-806 (2007) 
Dobigny G, Aniskin V, Volobouev V: Explosive chromosome evolution and speciation in the gerbil genus Taterillus (Rodentia, Gerbillinae): a case of two new cryptic species. Cytogenet Genome Res 96:117-124 (2002).

Dobigny G, Aniskin V, Granjon L, Cornette R, Volobouev V: Recent radiation in West African Taterillus (Rodentia, Gerbillinae): the concerted role of chromosome and climatic changes. Heredity 95:358-368 (2005).

Granjon L., Colangelo P, Tatard C, Colyn M, Dobigny G, Nicolas V: Intrageneric relationships within Gerbilliscus (Rodentia, Muridae, Gerbillinae), with characterization of an additional West African species. Zootaxa 3325: $1-25$ (2012).

Griffin M: A review of taxonomy and ecology of gerbilline rodents of the Central Namib Desert, with keys to the species (Rodentia: Muridae); in Seely MK (ed): Namib Ecology: 25 Years of Namib Research, pp 83-98 (Transvaal Museum, Pretoria 1990).

-Kovalskaya YM, Aniskin VM, Bogomolov PL, Surov AV, Tikhonov IA, et al: Karyotype reorganisation in the subtilis group of birch mice (Rodentia, Dipodidae, Sicista): unexpected taxonomic diversity within a limited distribution. Cytogenet Genome Res 132: 271-288 (2011).

Musser GG, Carleton MD: Superfamily Muroidea, in Wilson DE, Reeder DM (eds): Mammal Species of the World. A Taxonomic and Geographic Reference, pp 1210-1245 (Johns Hopkins University Press, Baltimore 2005).

Pavlinov IJA: Current concepts of gerbillid phylogeny and classification; in Denys C, Granjon L, Poulet A (eds): African Small Mammals. Paris: Colloquies et Seminaries, pp 141149 (IRD Editions, Paris 2001).
Perrin MR, Dempster ER, Downs CT: Gerbillurus paeba. Mamm Species 606:1-6 (1999a).

Perrin MR, Dempster ER, Downs CT: Gerbillurus tytonis. Mamm Species 607:1-4 (1999b).

Qumsiyeh MB: Chromosomal Evolution in the Rodent Family Gerbillidae. PhD thesis, Texas Tech University, Texas (1986a).

Qumsiyeh MB: Phylogenetic studies of the rodent family Gerbillidae: I. Chromosomal evolution in the Southern African complex. J Mamm 67: 680-692 (1986b).

Qumsiyeh MB: Pattern of heterochromatic variation and phylogeny in the rodent family Gerbillidae. Texas J Sci 40:63-70 (1988).

Qumsiyeh MB, Chesser RK: Rates of protein, chromosome and morphological evolution in four genera of Rhombomyine gerbils. Biochem Syst Ecol 16:89-103 (1988).

Qumsiyeh MB, Hamilton MJ, Schlitter DA: Problems in using Robertsonian rearrangements in determining monophyly: examples from the genera Tatera and Gerbillurus. Cytogenet Cell Genet 44:198-208 (1987).

Qumsiyeh MB, Hamilton MJ, Dempster ER, Baker RJ: Cytogenetics and systematics of the rodent genus Gerbillurus. J Mamm 72:89-96 (1991).

Rens W, Fu B, O'Brien PCM, Ferguson-Smith M: Cross-species chromosome painting. Nat Protoc 1:783-790 (2006).

Robinson TJ, Ropiquet A: Examination of hemiplasy, homoplasy and phylogenetic discordance in chromosomal evolution of the Bovidae. Syst Biol 60:439-450 (2011).
Robinson TJ, Ruiz-Herrera A, Avise JC: Hemiplasy and homoplasy in the karyotypic phylogenies of mammals. Proc Natl Acad Sci USA 105:14477-14481 (2008).

Rokas A, Holland PWH: Rare genomic changes as a tool for phylogenetics. Trend Ecol Evol 15: 454-459 (2000).

- Sannier J, Gerbault-Seureau M, Dutrillaux B, Richard FA: Conserved although very different karyotypes in Gliridae and Sciuridae and their contribution to chromosome signatures in Glires. Cytogenet Genome Res 134:51-63 (2011).

- Seabright M: A rapid banding technique for human chromosomes. Lancet 2:971-972 (1971)

-Sumner AT: A simple technique for demonstrating centromeric heterochromatin. Exp Cell Res 75:304-306 (1972).

Telenius H, Carter NP, Bebb CE, Nordenskjöld M, Ponder BAJ, Tunnacliffe A: Degenerate oligonucleotide-primed PCR: general amplification of target DNA by a single degenerate primer. Genomics 13:718-725 (1992).

Volobouev V, Aniskin VM, Sicard B, Dobigny G, Granjon L: Systematics and phylogeny of West African gerbils of the genus Gerbilliscus (Muridae: Gerbillidae) inferred from comparative G- and C-banding chromosome analyses. Cytogenet Genome Res 116:269281 (2007).

Yang F, Graphodatsky AS: Animal probes and Zoo-FISH; in Liehr T (ed): Fluorescence in situ Hybridization (FISH) Application Guide, pp 323-346 (Springer, Berlin 2009).

- Yang F, Trifonov V, Ng BL, Kosyakova N, Carter NP: Generation of paint probes by flow-sorted and microdissected chromosomes; in Liehr T (ed): Fluorescence in situ Hybridization (FISH) Application Guide, pp 35-52 (Springer, Berlin 2009). 